\title{
eJRIEPS
}

Ejournal de la recherche sur l'intervention en éducation physique et sport

11 | 2007

Varia

\section{L'expérience personnelle du professeur d'EPS, approche clinique et implications didactiques : une étude de cas d'une enseignante débutante}

Pablo Buznic-Bourgeacq, André Terrisse et Eric Margnes

\section{OpenEdition}

1 Journals

Édition électronique

URL : https://journals.openedition.org/ejrieps/6854

DOI : $10.4000 /$ ejrieps.6854

ISSN : 2105-0821

Éditeur

ELLIADD

Édition imprimée

Pagination : 20-38

Référence électronique

Pablo Buznic-Bourgeacq, André Terrisse et Eric Margnes, «L'expérience personnelle du professeur

d'EPS, approche clinique et implications didactiques : une étude de cas d'une enseignante

débutante », eJRIEPS [En ligne], 11 | 2007, mis en ligne le 01 janvier 2007, consulté le 29 octobre 2021.

URL : http://journals.openedition.org/ejrieps/6854 ; DOI : https://doi.org/10.4000/ejrieps.6854

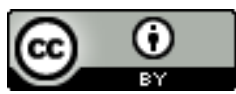

La revue eJRIEPS est mise à disposition selon les termes de la Creative Commons Attribution 4.0 International License. 
L'expérience personnelle du professeur d'EPS, approche clinique et implications

didactiques : une étude de cas d'une enseignante débutante

Pablo Buznic-Bourgeacq, André Terrisse, Eric Margnes

Université Paul Sabatier Toulouse III, EA3692, LEMME, AP3E, Toulouse, France.

\section{Résumé}

Cet article rend compte d'une recherche en didactique clinique de l'EPS s'intéressant à l'expérience personnelle du professeur d'EPS débutant dans les APSA qu'il a la charge d'enseigner. Au travers de l'étude du cas d'une enseignante stagiaire (PLC2), on tente d'identifier dans quelle mesure cette expérience personnelle oriente le contenu et la forme du processus de transmission des savoirs. Pour mener à bien cette étude, on s'attache à comparer son expérience et son enseignement effectif dans une activité qu'elle n'a jamais pratiquée (la lutte) et dans une autre qu'elle pratique depuis son enfance (la danse). Les outils conceptuels et méthodologiques nous permettant de décrire et interpréter cette comparaison s'inscrivent dans une position épistémologique particulière : la didactique clinique. On tente en effet de comprendre le fonctionnement des systèmes didactiques observés en articulant une analyse des contraintes didactiques et des contraintes internes qui structurent le sujet enseignant.

L'enseignant, dans sa classe, est inscrit dans un système de contraintes propre à l'activité de transmission des savoirs. Ces contraintes didactiques, et par extension les contraintes institutionnelles, tel que peut le problématiser Chevallard depuis une quinzaine d'années (1991), sont le centre des préoccupations de la recherche en didactique. Celle-ci tente de décrire et de comprendre la transmission des savoirs comme un système autonome régi par des déterminations qui lui sont spécifiques.

Toutefois, le processus de transmission, s'il peut être isolé, peut sembler gagner en compréhension à être pris en compte à partir du sujet qui le conduit, le sujet enseignant. C'est ce que tente la didactique clinique, impulsée par Terrisse (1994) en EPS (Education Physique et Sportive), en s'attachant à décrire et interpréter les phénomènes didactiques en les articulant avec le fonctionnement singulier du sujet enseignant. Car celui-ci rentre dans la classe avec un passé qui le lie aux savoirs, et détermine alors un système de 
contraintes intrinsèques au sujet enseignant (Blanchard-Laville, 2001) qui peut s'inscrire comme cause de certains phénomènes didactiques. Ce passé pourra être pris en compte comme un « déjà-là » (Terrisse, 1998), une éthique (Margnes, 2002), un « filtre » (Loizon, 2004), ou comme un rapport aux savoirs, indice d'une expérience personnelle (et corporelle) avec les savoirs d'une activité.

En effet, cet article s'intéresse au poids de l'expérience antérieure d'une enseignante dans une APSA sur son enseignement effectif en classe (Buznic-Bourgeacq, 2005). Car enseigner présuppose de savoir. Or, particulièrement en EPS, la multiplicité des objets de savoirs susceptibles d'avoir à être enseignés ne peut pas véritablement avoir été rencontrée par l'enseignant, compte tenu de la diversité des APSA constituant le champ social. Et, «si ce problème se pose dans d'autres disciplines, comme enseigner la géographie quand on est historien de formation ou la chimie quand on est physicien, il prend en EPS une importance plus affirmée du fait du statut du corps dans ce processus. » (Terrisse, Loizon, Buznic-Bourgeacq, 2005). Dans la mesure où le savoir en EPS est « incorporé », l'expérience de sa rencontre antérieure peut sembler fondamentale dans son enseignement.

Empiriquement, la recherche tente de comprendre dans quelle mesure l'expérience personnelle d'un enseignant débutant dans une activité physique influence le contenu et la forme de son enseignement effectif. II s'agit donc d'une analyse de pratiques effectives, qui s'intéresse au didactique ordinaire (Venturini, Amade-Escot, Terrisse, 2002), en empruntant son système descriptif principalement à la didactique des mathématiques, aux théorisations de Brousseau (1986) et Chevallard (1991), et qui porte son regard sur le sujet à partir des approches cliniques en sciences de l'éducation ayant un arrière-plan psychanalytique (Mosconi, Beillerot, Blanchard-Laville, 2000), ainsi que des prémisses de la théorisation en didactique clinique de l'EPS de l'équipe AP3E.

C'est donc un croisement entre l'étude synchronique du système didactique et l'examen diachronique du sujet enseignant, de son rapport aux savoirs, à la fois processus et produit de son histoire, qui spécifie la perspective clinique en didactique. Ainsi, d'un point de vue méthodologique, les trois temps usuels de l'analyse didactique (analyse a priori, observation in situ, analyse a posteriori) sont complétés par une analyse clinique de l'expérience antérieure de l'enseignant, permettant alors des inférences sur les causes de certains phénomènes didactiques. En s'appuyant sur l'étude comparative du cas d'une enseignante d'EPS débutante, menant un enseignement de sa spécialité, la danse, et celui d'une activité qui lui est particulièrement étrangère, la lutte, on présente ici d'abord 
une tentative d'identification et de structuration de son rapport aux savoirs. Ensuite, à partir de deux corpus de séances, nous examinons dans quelle mesure ce rapport fonde le contenu de son enseignement, puis décrire et interpréter, à partir de son discours après-coup, comment une différence d'expérience détermine la forme de l'enseignement mené, dans sa gestion du contrat didactique.

\section{L'expérience et le rapport de l'enseignant aux savoirs}

L'expérience personnelle de l'enseignante est interprétée en tant que rapport aux savoirs. Ainsi, cette problématisation en terme de lien entre un sujet et différents objets de savoir nous permet de caractériser cette expérience dans une perspective didactique. Sachant qu'il existe plusieurs approches théoriques de cette problématique, nous nous inscrivons dans la complémentarité de deux d'entre elles : les approches anthropologique/didactique et clinique ${ }^{1}$.

L'approche anthropologique du didactique de Chevallard recourt à la question du rapport aux savoirs pour l'inscrire dans une problématique institutionnelle. Définissant une personne comme «l'émergent d'un complexe d'assujettissements institutionnels », Chevallard (1992) identifie la manière personnelle de connaître un objet de savoir, soit le rapport personnel au savoir, comme le produit de sélections, de restrictions, de concessions, d'oublis faits à propos des divers rapports institutionnels auxquels le sujet a été confronté. Ce sont ici les institutions, au sens anthropologique du terme, largement exploité en didactique, qui structurent le rapport du sujet aux savoirs. En revanche, dans la perspective clinique, inspirée de la psychanalyse (Blanchard-Laville, 2002), la problématique du rapport aux savoirs ne se confond pas avec la précédente. Ici le sujet est considéré comme responsable et comme source irréductible de son rapport aux savoirs. Ainsi, au-delà des assujettissements qui structurent le rapport du sujet aux savoirs, on considère que ce rapport ressort aussi d'un processus intrinsèque constitué tout au long de son expérience subjective. Car les différents objets de savoir, rencontrés et créés dans l'expérience du sujet, ont pu être source de plaisir, de déplaisir ou d'angoisse, de sorte que son rapport aux savoirs s'inscrit aussi dans une dialectique désir/défense, par laquelle chaque savoir est porteur d'un sens spécifique pour le sujet. On s'intéresse donc ici à l'histoire du sujet et aux divers objets institués susceptibles d'avoir fait travailler ses désirs (et par opposition complémentaire ses défenses), et par là

\footnotetext{
${ }^{1}$ Pour un autre exemple, assez peu courant, de complémentarité entre les approches didactique et clinique du rapport au savoir on pourra lire Berdot, Blanchard-Laville, Bronner (2000).
} 
même, le regard est porté sur l'investissement spécifique du sujet dans ces objets de savoir comme fondement d'un "processus créateur de savoir », soit d'un rapport au savoir (Beillerot, 2000).

II ne s'agit pas là de tenter une synthèse, porteuse de confusions épistémologiques, mais bien de tenir compte de deux dimensions irréductibles du sujet afin de rendre compte du rapport qu'il entretient avec les divers objets de savoir : le sujet est à la fois institutionnel et pulsionnel. Cette position s'inscrit dans une tradition scientifique de « l'interstructuration du sujet et des institutions », dans la continuité des options de Wallon et de Malrieu (Curie, Tap, 1983).

Afin de modéliser ce rapport de l'enseignante aux savoirs, on a donc pris en compte ces deux aspects. Mais, ayant déjà en arrière-plan l'idée d'un processus de conversion de l'expérience du sujet en objets de savoirs enseignés, on a aussi, pour cristalliser une étape de cette conversion et suivre à la trace certains invariants, distingué deux formes de ce rapport. L'une que nous avons nommée rapport expérientiel aux savoirs, relevant de ce qui lie le savoir à l'histoire du sujet, qui ressort de son expérience brute et qui ne suscite pas une attitude réflexive sur le savoir mais plutôt sur sa propre histoire. On y a accès par des entretiens de type « histoire de vie » (Pineau, Legrand, 1996), par lesquels on examine les institutions et les investissements subjectifs au travers desquels l'enseignante a établi son propre rapport aux savoirs. L'autre que nous avons nommée rapports épistémologique et didactique aux savoirs, concepts proches de «l'épistémologie professionnelle » de Brousseau (1998), qui ressortent des formalisations au travers desquelles l'enseignante conçoit l'activité et son enseignement. On les identifie par des entretiens semi-structurés permettant d'établir une énumération et un système de relations entre les savoirs qui structurent chaque APS pour l'enseignante.

On présente ici une synthèse des principaux éléments qui constituent son rapport aux savoirs, à partir de son discours et d'une brève systématisation. 
Tableau I. Le rapport de l'enseignante aux savoirs

\begin{tabular}{|c|c|c|c|}
\hline & \multicolumn{2}{|l|}{ Expérientiel } & \multirow{2}{*}{$\begin{array}{l}\text { Formalisation } \\
\text { Epistémologique et Didactique }\end{array}$} \\
\hline & Institutions & Désir/Défense & \\
\hline Lutte & $\begin{array}{l}\text { - «j'ai jamais } \\
\text { fait de lutte » } \\
\text { - «j'ai fait du } \\
\text { judo en } \\
\text { STAPS, en } \\
\text { première } \\
\text { année »; } \\
\text { “j'ai vu faire } \\
\text { un spécialiste } \\
\text { judo en EPS » }\end{array}$ & $\begin{array}{l}\text { - “J'ai détesté »; “me battre } \\
\text { moi, c'est pas trop ça » } \\
\text { - «j'avais beaucoup de } \\
\text { blessures »; "les filles, elles } \\
\text { étaient bien plus taillées que } \\
\text { moi » } \\
\text { - «j'aimais pas ça quoi, c'était de } \\
\text { l'apprentissage de gestes »; “ça } \\
\text { ne me plaisait pas, parce qu'il n'y } \\
\text { a aucune logique par rapport au } \\
\text { sens de l'activité » }\end{array}$ & $\begin{array}{l}\text { - Formalisation confuse entre savoirs } \\
\text { issus de la lutte et issus du judo } \\
\text { (techniques d'immobilisation) } \\
\text { - Deux types de savoirs au centre de la } \\
\text { structure de l'activité : savoirs } \\
\text { sécuritaires (chutes) et savoirs } \\
\text { tactiques/stratégiques } \\
\text { d'action/réaction) } \\
\text {-Présence de quelques savoirs } \\
\text { techniques, toujours dans une } \\
\text { perspective d'utilisation raisonnée en } \\
\text { combat }\end{array}$ \\
\hline Danse & 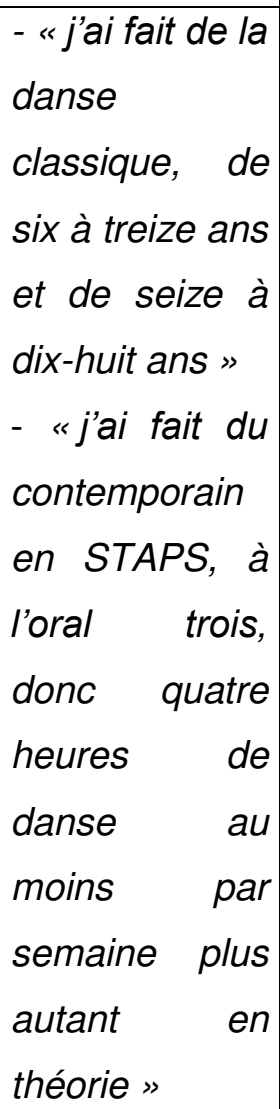 & $\begin{array}{l}\text { - « (la contemporaine) a } \\
\text { beaucoup plus de sens qu'une } \\
\text { chorégraphie carrée... ça c'est } \\
\text { que de la forme »; " mais il y a } \\
\text { des fois, ça ne ressemble plus } \\
\text { trop à de la danse » } \\
\text { - «il a fallu tout changer... ça a } \\
\text { été plutôt un frein »; " quand tu } \\
\text { passes au contemporain, t'es } \\
\text { bloqué quoi... parce que tu sais } \\
\text { pas t'exprimer » } \\
\text { - « c'est une chorégraphie où ça } \\
\text { sort de toi et... oui, il y a } \\
\text { beaucoup plus de sens » }\end{array}$ & $\begin{array}{l}\text { - Une APS structurée par une diversité } \\
\text { de savoirs précis (temps, espace, } \\
\text { énergie, relation aux autres, différents } \\
\text { rôles...) } \\
\text { - Clivage fondamental surplombant la } \\
\text { structure de l'activité : entre rigueur } \\
\text { technico-esthétique et libre } \\
\text { interprétation } \\
\text { - Difficultés du passage de la danse } \\
\text { classique à la contemporaine projetée } \\
\text { sur les élèves filles danseuses } \\
\text { classiques } \\
\text { - Suprématie de la libre interprétation } \\
\text { qui englobe finalement la technique à } \\
\text { son service }\end{array}$ \\
\hline
\end{tabular}

Cette présentation du rapport de l'enseignante aux savoirs permet de situer les différents objets de savoirs qui le constituent ainsi que les rapports institutionnels susceptibles de le 
fonder et les investissements subjectifs de l'enseignante dans ces divers objets. II s'agit donc du rapport aux savoirs dans une approche didactique clinique qui se propose comme un outil de compréhension des pratiques enseignantes. Sa construction s'élabore selon une structure (rapport expérientiel, assujettissements institutionnels, investissements pulsionnels et défensifs, formalisations épistémologique et didactique) dans laquelle le cas de notre enseignante trouve sa place singulière.

II reste à examiner ce que ce rapport aux savoirs permet de comprendre de sa pratique.

\section{Implications didactiques du rapport de l'enseignant aux savoirs : deux corpus d'analyse}

On présente ici deux corpus, un en lutte et un en danse, constitués d'épisodes d'une dizaine de minutes, issus de la dernière partie des deuxièmes séances observées des cycles d'enseignement de chaque activité. Les retranscriptions relèvent des interactions de l'enseignante avec les élèves en pleine activité. Ces extraits servent ainsi d'exemple au cadre interprétatif qui soutient notre étude comparative ; leur choix a été effectué en vertu de leur caractère exhaustif par rapport à la diversité de nos conclusions, établies à partir des corpus complets des quatre séances analysées.

TABLEAU II. Corpus 1, extrait de la deuxième séance de lutte

\section{LUTTE}

Situation : Les élèves sont par quatre (deux arbitres, deux combattants). Ils font des combats au sol de trois minutes. Ils partent à genoux et doivent immobiliser l'adversaire au sol dix secondes. S'ils immobilisent l'adversaire avec une des prises précédemment apprises (Hon gesa gatame et Kuzure gesa gatame) ils marquent dix points ; s'il s'agit d'une autre prise ils en marquent cinq. Le but est d'avoir le plus de points.

- 1h05min23s: Deux élèves à genoux tentent de s'amener au sol et n'y arrivent pas. Elles sont assez éloignées et n'ont pas de saisies efficaces entre elles. L'enseignante les regarde longuement puis intervient. - Verbatim : P. « Hé... les filles, stop... vous ne vous accrochez pas bien... accroche-la là et là... (en guidant son bras sous l'aisselle de son adversaire vers son omoplate et en lui montrant, pour l'autre bras, le bras gauche de son adversaire)... (les élèves reprennent le combat accrochées comme demandé)... voilà, là c'est bien... »

- 1h07min54s : Deux élèves combattent et l'enseignante s'approche d'eux, l'un des élèves l'interpelle, car il n'arrive pas à saisir assez fermement son adversaire pour le déséquilibrer (il a un bras derrière le dos de son adversaire mais il a la main ouverte sans véritable appui et il n'est pas collé à lui)

- Verbatim : L'élève : « Eh, madame, j'arrive pas à le tenir... ça m'énerve »

P. «Attend j'ai pas vu... (elle se rapproche des élèves)... prend-le là (en guidant son bras, qui était posé sur le bas du dos, vers l'omoplate de son adversaire)... là. " (l'élève se tient comme demandé et semble mieux résister à son adversaire, sans tout de même vraiment réussir à l'amener au sol)

- 1h14min33s : À la fin des trois minutes d'un combat, l'enseignante arrête ses élèves, leur demande de l'attention; les élèves s'assoient autour du tatami

- Verbatim : P. « Dernier match... Alors juste... vous prenez... certains prennent mal leur adversaire... N'estce pas les filles... vous essaierez de le prendre aux épaules... ou par exemple au bas... et vous le prenez derrière (en mimant un grand cercle avec ses bras)... d'accord... mais vous ne le prenez pas sur une toute petite surface sinon vous aurez du mal à le projeter (en mimant un très petit cercle avec ses bras). » 
TABLEAU III. Corpus 2, extrait de la deuxième séance de danse

\section{DANSE}

Situation : Les élèves sont par quatre. Ils doivent effectuer une chorégraphie (sans musique) de quatre fois huit temps, avec pour thème "le cauchemar " en y intégrant des éléments techniques imposés (un déplacement, une chute, un passage au sol, un tour et un saut). Ils ont à leur portée des feuilles sur lesquelles différents éléments sont proposés.

- 1h04min44s: Un groupe d'élèves montre sa chorégraphie à l'enseignante, qui ne dure que deux fois huit temps. Dans la chorégraphie, le saut et le tour sont présents mais peu visibles. De plus, leur chorégraphie relève principalement d'un aller-retour sur la scène, les quatre élèves en même temps, alignés et effectuant exactement la même chose. Ça ne plaît pas à l'enseignante qui intervient.

- Verbatim : P. « C'est fini ? Alors, ça fait que deux fois huit temps, il vous manque la moitié... Bon... Alors, il y a quelque chose qui me dérange un peu... c'est que déjà... le tour, le saut, c'est pas du tout précis, donc on ne voit pas... sauf Mat qui fait un tour au début, on l'a vu... les autres... on n'a pas vu... »

Un élève : "Mais là-bas, on a fait comme ça » (en montrant le bout de la scène et en mimant l'impulsion d'un saut)

P. «Alors, ce que je veux, c'est que... vous êtes plus précis... ça veut dire que... si on se déplace, on se déplace... si on veut tourner, on s'arrête, et après on tourne... mais, il faut qu'on voit les choses... parce que là vous courez, vous êtes là, vous courez, vous courez, vous courez (en courant comme eux jusqu'au bout de la scène)... vous faîtes un truc comme ça (en faisant un tour rapide et déséquilibré sans s'arrêter)... et c'est reparti (en continuant de courir)... non, moi je veux pas ça... moi je veux (elle se replace de l'autre côté de la scène)... on court (elle recommence à courir comme précédemment)... on court, puis on s'arrête (elle s'arrête)... puis là on tourne (en faisant un tour équilibré)... on s'arrête, d'accord... Donc, autre chose... vous êtes quatre... c'est pas quand-même super original d'être un, deux, trois, quatre (en pointant les élèves alignés d'un côté de la scène)... et de faire quatre comme ça (en montrant avec ses bras le trajet des élèves traversant la scène)... et quatre comme ça (en montrant le trajet inverse)... dans le style, vous pouvez faire un déplacement en ligne droite, et après on change... on peut faire un cercle (en montrant un trajet circulaire avec ses bras)... par exemple, si vous êtes dans l'armée, si vous avez peur et tout, vous pouvez vous regrouper (en faisant le dos rond et en se cachant derrière ses mains), faire un cercle, rouler sur vous-même, vous cacher, des choses comme ça... II vous manque deux fois huit temps, alors essayez d'en rajouter... donc, le début, vous pouvez commencer comme ça... bon... allez on refait le début... (les élèves se placent comme au début de leur chorégraphie)... là, vous dormez... voilà... et comment on sait si c'est un cauchemar ? »

Un élève : «On fait comme ça » (en faisant semblant de dormir en se débattant)

P. «Voilà... et là vous prenez du temps... (les élèves se lèvent)... et puis au bout (en se rapprochant de l'autre côté de la scène)... vous pouvez arriver... et vous accroupir (en s'accroupissant)... Vous voyez, il faut faire des choses en plus du tour, saut et chute... ok " (les élèves se mettent à réfléchir sur des éléments à placer dans leur chorégraphie)

Ces corpus servent ici de fondement à l'analyse de lien entre le contenu, la forme de l'enseignement mené et l'expérience personnelle de l'enseignante.

\section{Implications didactiques sur le contenu}

\subsection{Sur le contenu de l'enseignement : la conversion didactique}

Les interrogations du chercheur sur le contenu de l'enseignement renvoient, au moins depuis Chevallard (1991), à la problématique de la transposition didactique. Le chercheur en didactique se pose la question de la référence des savoirs enseignés (Terrisse, 2001).

Si l'intention d'enseigner transforme inévitablement les objets pris par cette intention, demeure la question de l'origine, toujours « ailleurs » mais seule source de légitimité, de ces objets. Reste que cette légitimité n'est jamais univoque, et particulièrement en EPS, «la multiplicité des références est son histoire même » (Terrisse, 2001). Tout au long du 
XXème siècle, au travers de nombreuses luttes de pouvoirs entre des plausibles et désireux noosphériens, l'EPS s'est référée à diverses institutions légitimantes : l'armée, la médecine, les jeux traditionnels, le sport de compétition... Encore aujourd'hui, si le sport fédéral est la référence fondamentale de l'EPS, certaines pratiques d'entretien physique ou encore les jeux du cirque tendent à trouver une certaine légitimité dans l'enseignement scolaire. Et au-delà de cette diversité institutionnelle qui détermine la légitimité des objets enseignés en EPS, il faut retenir que les pratiques sociales qui valent pour référence ressortent d'une multiplicité d'APS, conçues et pratiquées selon diverses finalités: compétitive, ludique, hygiénique, et selon diverses conceptions du sport : culturaliste, socialisateur, développemental, etc.

C'est donc cette diversité de modalités de pratiques, et par là même de références, qui nous interroge ici. Considérant alors qu'aux portes de la classe seul l'enseignant, relais institutionnel scolaire, apporte la légitimité des objets de savoir auxquels les élèves seront confrontés, le questionnement sur le passage du contenu de son expérience à celui de son enseignement devient fondamental pour le chercheur. Car, «le magma didactique, c'est bien cet ensemble d'objets autres (alien) auxquels l'élève n'établit rapport que par l'intermédiaire (du rapport) de la maîtresse » (Sensevy, 1997).

Alors, la didactique clinique établit le postulat d'un processus, celui de conversion didactique de l'expérience de l'enseignant en contenu d'enseignement. Il questionne celui de transposition didactique en dépassant sa seule inscription dans une problématique purement institutionnelle. Reste à l'identifier au travers d'études de cas afin de lui donner consistance.

3. 2. La conversion didactique : le cas de l'enseignante

Méthodologiquement, il s'agit d'un travail comparatif entre le contenu de son rapport aux savoirs et le contenu de son enseignement. Deux approches le définissent: l'une qualitative qui, à partir d'épisodes précis, tente d'identifier les traces de la structure de son rapport aux savoirs dans sa transmission (tel que l'on va en montrer ici un exemple), l'autre plus quantitative qui examine pour chaque mise en jeu par l'enseignante d'un objet de savoir s'il constitue a priori son rapport aux savoirs.

Dans le corpus LUTTE présenté ici, on peut ainsi s'arrêter sur deux points. Premièrement, on peut voir que le principal objet de savoir en jeu dans la situation relève d'un Savoir utiliser un technique d'immobilisation précise (Hon gesa gatame ou Kuzure gesa gatame) en situation de combat. Rappelons que ces techniques sont issues du judo et que l'activité lutte diffère du judo de part sa logique interne, dans la mesure où la victoire y est acquise 
par le simple contact des épaules de son adversaire avec le sol. Encore, cet enjeu de savoir est contrôlé par une variable didactique (le score différent selon la technique utilisée), qui est là pour rappeler - de manière ambiguë - aux élèves que ces prises ne sont pas une finalité en elles-mêmes mais gardent une «logique par rapport au sens de l'activité ». Deuxièmement, les trois interventions de l'enseignante mettent en jeu un nouvel objet de savoir qui ressort d'un Savoir maintenir son adversaire par des saisies efficaces permettant de le déséquilibrer ("vous ne vous accrochez pas bien, accroche-la là et là »; “prend-le là »; “ certain prennent mal leur adversaire »). Ainsi, a priori, cet objet ne semble pas constituer son rapport aux savoirs de l'activité lutte, mais émerge par sa confrontation avec l'activité adaptative des élèves; et cette émergence semble alors révéler une forme d'adaptation de l'enseignante elle-même aux savoirs de la lutte qui lui sont jusque là inconnus. Quantitativement, 47\% des objets de savoir mis en jeu par l'enseignante en lutte ne sont pas identifiables dans l'analyse antérieure de son rapport aux savoirs et ne semblent être générés que par leur nécessité face au vif des séances.

En revanche en danse, seuls $2 \%$ n'y sont pas identifiables. Ainsi, dans le corpus DANSE, on peut voir qu'une grande diversité d'objets de savoir est mise en jeu dans l'épisode, à propos de la gestion du temps dans la chorégraphie (« II vous manque deux fois huit temps, alors essayez d'en rajouter »), des aspects esthétiques des éléments techniques (équilibre et distinction des éléments: "ce que je veux, c'est que... vous êtes plus précis ») et de la réalisation collective ( “c'est pas quand-même super original d'être un, deux, trois, quatre »), de l'interprétation expressive du thème ( si vous avez peur et tout, vous pouvez vous regrouper, faire un cercle... »). On verra ultérieurement que la gestion du contrat didactique trouve sa spécification dans cette diversité qui semble s'imposer à elle. On peut notamment remarquer comment la rigueur technico-esthétique («il y a quelques chose qui me dérange un peu... ") et l'interprétation expressive (" et comment on sait si c'est un cauchemar ?») lui sont essentielles et constituent le fond de son intervention.

II semble ainsi que le processus de conversion didactique que l'on a postulé trouve ici une certaine épaisseur. On voit en effet que dans une activité connue la multiplicité des savoirs qui structurent son rapport aux savoirs est enseignée presque inévitablement face à l'activité des élèves, révélant par là même ici les traces des différentes institutions (danse classique et contemporaine) auxquelles l'enseignante a pu être assujettie. De même, dans une activité inconnue, la conversion s'établie à partir d'une autre activité formant son expérience antérieure, mais garde aussi les traces d'une défense établie à propos d'une 
expérience déplaisante (la confrontation à l'enseignement d'un judo techniciste lui semblant dénué de sens). Toutefois, l'absence d'expérience antérieure semble conduire à l'émergence d'objets de savoirs qui ne trouvent pas leur source dans l'expérience de l'enseignante mais qui semblent être générés de sa confrontation à l'activité des élèves, révélatrice de son manque à savoir. Ces émergences ressemblent à une adaptation et jettent les premiers éléments de compréhension du rapport entre expérience dans l'activité enseignée et forme de son enseignement.

\section{Implications didactiques sur la forme}

Le contrat didactique constitue un outil descripteur de la contingence de l'enseignement (Terrisse, Léziart, 1999).

4. 1. Sur la forme de l'enseignement : la gestion du contrat didactique.

Ce contrat permet de rendre compte du jeu de responsabilités sous-jacent au processus de transmission des savoirs. Pour une analyse riche et complète de la problématique du contrat didactique, on peut consulter Sarrazy (1995). On notera que le concept est né dans la constitution de la Théorie des Situations de Brousseau (1986). II s'articule donc et ne trouve son véritable sens qu'en référence au processus de dévolution inhérent à la perspective brousseauiste de la transmission des savoirs.

L'intention est ici de comprendre comment l'expérience du sujet enseignant détermine la gestion de ce jeu de responsabilités, soit de la contingence du processus, ceci afin de mettre en avant le poids de sa subjectivité sur la construction du contrat et, par là même, la pertinence d'une didactique clinique comme approche explicative des pratiques d'enseignement. La gestion de la contingence du processus de transmission, tel qu'elle peut être décrite par le contrat didactique, peut être identifiée au travers de deux aspects sous-jacents au jeu de responsabilités établi dans la classe : la chronogenèse et la topogenèse (Chevallard, 1991).

Premièrement, "dans le processus didactique, le savoir est un savoir-temps " (Sensevy, Quilio, 2002). La transmission des savoirs suit une progression temporelle et, à chaque instant, "la base des objets et des rapports institutionnels à ces objets ayant antérieurement émergé " (Chevallard, 1989) compose de fond de contrat de chaque situation. Chevallard (1991) caractérise différentes formes d'objets dans l'espace didactique : objets de savoir, objets paramathématiques et protomathématiques. On ne peut pas considérer exactement ces objets en EPS, car la nature du savoir en jeu y est fort différente. Ainsi, ce sont principalement les objets de savoir qui constituent ici le fond 
de l'analyse de la chronogenèse. Néanmoins, l'enseignant occupe une place fondamentale dans ce processus. II est celui qui marque le contrat des nouveaux objets sur lesquels il va reposer. C'est le rôle qu'il assume alors particulièrement au travers des phases d'institutionnalisation: faire élaborer un code symbolique, «un symbolique enraciné dans l'historicité du processus de rationalisation. (Car), après-coup vous construisez une histoire du monde. L'institutionnalisation est une phase de réorganisation, de construction en commun » (Brousseau, in Terrisse, Léziart, 1999). Nos interrogations reposent alors ici sur la spécificité de l'activité de l'enseignant dans la construction de cet ancrage institutionnel des savoirs. II s'agit d'examiner dans quelle mesure son expérience antérieure détermine cet ancrage et, par là même, le degré de « fermeture » du contrat didactique.

Deuxièmement, à chaque instant de la chronogenèse, chacun, professeur et élèves, occupe une place définie par un ensemble de gestes possibles et par un degré de responsabilité vis-à-vis de l'émergence des objets de savoir. Cette «partition déterminée de l'espace symbolique » (Sensevy, Quilio, 2002) établit la position symbolique de chacun et peut être identifiée par le processus de topogenèse des savoirs. Ainsi, lorsqu'un problème a été dévolué aux élèves, qu'ils sont en pleine activité d'adaptation et de recherche, l'enseignant a pour rôle, tout en laissant les élèves s'approprier seuls le savoir, de maintenir la relation didactique en participant à la gestion des obstacles des élèves. C'est donc pour une grande part dans ces interactions que se joue la genèse du savoir, particulièrement en EPS (Loquet, Marsenach, Amade-Escot, 2005). Alors, dans ces phases, l'enseignant peut reprendre à son compte certaines responsabilités qui étaient dévoluées jusque là aux élèves, mais il peut le faire de différentes manières. II s'agit alors ici d'examiner comment, dans ces interactions, l'enseignant prend à son compte l'émergence du savoir, en le rendant davantage visible aux élèves, en le « donnant à voir », c'est-à-dire au travers de différentes «procédures ostensives locales » (Salin, 2002). L'intérêt porte alors sur le poids de son expérience antérieure sur l'utilisation de ces procédures.

On décrit alors ici, de manière comparative en lutte et en danse, les spécificités de ces deux aspects de la gestion du contrat pour le cas de l'enseignante : la récurrence des phases d'institutionnalisation et des savoirs officiels (savoirs qui ont déjà été institutionnalisés et ont donc un statut officiel pour l'institution classe) d'une part, et la récurrence des différentes procédures ostensives d'autre part. Et au-delà de ces descriptions, il s'agit de tenter d'interpréter les causes de ces spécificités, à partir de la 
perspective didactique clinique. Ayant déjà identifié son rapport aux savoirs des deux activités pour en faire un élément d'interprétation, cette approche s'appuie aussi sur un procédé méthodologique spécifique: l'entretien d'après-coup (Terrisse, 2000). II s'agit d'entretiens effectués plusieurs mois après les observations consistant à donner la parole au sujet à propos des raisons de ses actes passés et, notamment, à identifier les formes de son implication subjective dans ses interventions. Ces entretiens d'après-coup ont pour fondement théorique l'après-coup freudien. «D'emblée, Freud a marqué que le sujet remanie après-coup les évènements passés et que c'est ce remaniement qui leur confère un sens » (Laplanche, Pontalis, 1967). II a notamment été repris en didactique par Chevallard (1991) pour caractériser une phase nécessaire à l'appropriation des savoirs, celle de remaniement. Ainsi, le poids du sujet enseignant et de son expérience sur la gestion du contrat didactique pourra être identifié, révélant ainsi les contraintes intrinsèques qui pèsent sur le sujet pris dans le didactique.

4. 2. La chronogénèse du savoir, le rapport à la contingence : le cas de l'enseignante Dans le corpus LUTTE, on l'a explicité, de nouveaux savoirs émergent du vif des séances et semblent faire apparaître une adaptation de l'enseignante aux savoirs de l'activité. On peut ainsi remarquer que dès sa première intervention, par l'observation de deux élèves combattant n'arrivant pas à se saisir assez efficacement pour se déséquilibrer, elle met en jeu très succinctement un nouveau savoir à propos des saisies et du placement du corps auquel elles conduisent. Elle poursuit alors pour intervenir auprès de deux autres élèves, mettant en jeu identiquement le même savoir. Puis, dès que le tour de combat se termine, elle en profite pour s'empresser d'arrêter l'activité des élèves afin d'institutionnaliser cet objet de savoir, l'édifiant alors comme un objet reconnu par l'institution classe et ainsi un constituant explicite du contrat didactique. En fait, cet ancrage institutionnel va être récurrent durant tout son enseignement. Quantitativement, sur deux séances, on peut compter dix institutionnalisations pour vingt-deux interventions ponctuelles et dans ces interventions ponctuelles, on compte dix-huit fois la mise en jeu d'un objet de savoir officiel contre seulement quatre nouveaux savoirs.

En revanche, en danse, on ne compte que quatre institutionnalisations pour trente-six interventions ponctuelles, et dans ces interventions, dix-huit savoirs officiels sont mis en jeu contre vingt-huit nouveaux savoirs. On voit ainsi, par exemple, dans le corpus DANSE, qu'une diversité d'objets de savoir est mise en jeu, et ils ne seront que peu à prendre une place explicite dans les fondements institutionnels du contrat. Tous ces savoirs ont une 
place ambiguë dans le jeu de responsabilités établi à propos des savoirs et forment ainsi un contrat didactique aux contours flous.

II semble alors que les enseignements de lutte et de danse soient fondés sur deux formes de gestion du contrat didactique, l'une s'appuyant sur une reconstruction permanente et explicite du contrat par un fort ancrage institutionnel, l'autre s'établissant sur un fond de contrat peu cadré et diversifié.

D'où vient alors cette différence ? En lutte, l'adaptation de l'enseignante aux savoirs trouve une forme spécifique. Toute tentative de réponse à un obstacle d'un élève s'appuie presque toujours sur un objet de savoirs déjà institutionnalisé, et lorsque le maigre éventail de ces savoirs ne suffit pas, elle tente parfois de trouver une solution par un nouvel objet. Mais, chaque nouveauté se voit prendre immédiatement un statut institutionnel. L'identification des obstacles des élèves semble moins servir à leur donner des réponses singulières qu'à structurer le fond de contrat didactique de la classe. S'adaptant, elle ne peut s'empêcher de figer l'avancée du savoir, de l'écrire institutionnellement. Son discours après-coup peut permettre de comprendre cette spécificité. En effet, elle semble se trouver limitée d'un point de vue didactique : «j'étais plutôt papillon... sur le tatami à gérer ma classe... mais au niveau des contenus, un peu léger quand-même». Et cette impuissance semble être pour elle le sceau de son illégitimité didactique : «le problème, c'est que des fois, tu ne maîtrises pas du tout et... le problème, c'est que ça, ils le savent, ils le ressentent». Ainsi, faible d'une autorité didactique ambiguë, l'ancrage institutionnalisant de son enseignement semble être une voie de légitimation pour ellemême : «ça fait un peu... pour pouvoir se dire... j'ai trouvé quelque chose à leur dire... alors autant en parler à tout le monde... au moins ça sera posé... ». La contingence du processus lui est impossible à supporter et elle se réfugie alors dans des inscriptions officielles des savoirs, protégée ainsi par l'institution (Beillerot, 1996), lui permettant d'occuper sa position symbolique dans l'espace didactique, celle de « sujet supposé savoir » (Lacan, 1966 ; Chevallard, 1991), position vers laquelle tous les transferts des élèves sont dirigés et qu'il s'agit alors, tant bien que mal, d'assumer.

En revanche, en danse, elle ne craint pas cette contingence. Elle peut assumer pleinement cette position symbolique sans avoir à se dissimuler dans des refuges institutionnels. Au contraire, son expérience antérieure lui garantit l'évitement d'une illégitimité didactique perçue. Ainsi, ses interventions dépassent largement les cadres explicites du contrat didactique et sa confrontation à la contingence la conduit à mettre en jeu une diversité de savoirs qui troublent les frontières du jeu de responsabilités en 
vigueur. Cette diversité semble ainsi s'imposer à elle, comme une réponse à l'insupportable maladresse de l'activité des élèves. " Des fois ça ne ressemble plus trop à de la danse, et ça... ça m'embête un peu». Une autre approche de l'analyse des pratiques pourrait renforcer ces conclusions, mais n'ayant pas été conduite ici de manière systématique elle ne peut être valablement présentée. II s'agit d'un analyse du discours effectif de l'enseignante durant les séances, à propos de son implication dans ce discours. On peut ainsi remarquer dans le corpus DANSE que plusieurs phrases de l'enseignante à propos des contenus d'enseignement commencent par des marqueurs de son implication personnelle : «Alors il y a quelque chose qui me dérange un peu... »; «Alors ce que je veux... ». On y verrait sûrement encore les marques de sa proximité avec l'activité.

On constate donc que le sujet enseignant, compte tenu de son expérience antérieure dans l'activité enseignée, pèse sur les aspects chronogénétiques de la gestion du contrat ; ceci dans la mesure où cette expérience détermine les possibilités du sujet à assumer sa position de «sujet supposé savoir » et, par conséquent, le rapport qu'il établit à la contingence du processus de transmission.

4. 3. La topogénèse du savoir, les figures de prise de responsabilité : le cas de l'enseignante

Dans le corpus LUTTE, lors de ses interventions ponctuelles, l'enseignante reprend à son compte certaines responsabilités vis-à-vis de l'émergence du savoir. Elle « donne à voir » du savoir par une manipulation du corps des élèves, en guidant et dirigeant leurs bras, reprenant ainsi à sa charge la genèse des éléments moteurs du savoir. Dans le corpus $D A N S E$, elle reprend aussi certaines responsabilités, mais d'une manière différente. Elle montre par son discours certains déterminants des savoirs, comme la nécessité de l'équilibre dans la réalisation des éléments techniques ou de la diversification collective des déplacements. Mais surtout elle montre du savoir par l'intermédiaire de son corps, par des démonstrations, en mettant en œuvre les savoirs, comme les éléments techniques équilibrés et distincts ou l'interprétation expressive de la peur, mais aussi les « manques de savoir », comme les éléments techniques déséquilibrés et confus. Ainsi, en danse, lorsqu'elle intervient, elle reprend sous sa responsabilité la genèse du savoir dans sa globalité, tel qu'il peut être compris, vu et réalisé.

L'intérêt porte donc ici sur ces différentes formes de procédures ostensives qui scandent les cours lors des interventions ponctuelles de l'enseignante. On les identifie alors selon les responsabilités que l'enseignante reprend à sa charge dans l'émergence du savoir. De cette identification ressortent notamment trois figures de procédures ostensives, telles 
qu'on a pu les décrire à partir des corpus : l'ostension directe physique, c'est-à-dire la démonstration, l'ostension directe verbale, soit l'explication par le discours, l'ostension physique privée, soit la manipulation du corps de l'élève. Ainsi, quantitativement, en lutte comme en danse, son enseignement s'appuie fortement sur les deux dernières : quinze fois sur vingt-deux interventions au total en lutte et vingt-deux fois sur quarante-six en danse. Cependant, la démonstration, elle, est presque absente en lutte (trois fois) alors qu'elle est particulièrement récurrente en danse (dix-neuf fois).

II semble ainsi que dans le contrat didactique, l'enseignante, après voir dévolué un problème aux élèves, reprenne largement à son compte certaines responsabilités. Mais la démonstration du savoir dans sa globalité et son intermédiaire corporel sont laissés à l'enseignement de la danse. Il y a des formes de responsabilités qui échappent à l'enseignante en lutte.

En effet, la démonstration en lutte n'est que peu envisageable : «Tu ne montres pas parce que t'es flag (pris en flagrant délit), donc tu te discrédites... », nous dit-elle aprèscoup. L'intermédiaire corporel de ce type de procédure ne peut que la placer dans un flagrant délit de non-savoir. Car, « ils le savent... ils le ressentent ». Or, encore une fois, sa position symbolique ne permet pas ce non-savoir, puisque dans l'espace didactique elle est le «sujet supposé savoir». Alors ne pouvant assumer sa position par l'intermédiaire de performances motrices, elle ne peut que tenter de la conforter en « donnant à voir » du savoir à partir du corps de l'élève ou d'une explication des éléments structurels de ce savoir : «du coup, tu dois te servir d'autres outils ${ }^{2}$. En revanche, en danse, la récurrence de la démonstration semble être un moyen d'assumer sa position symbolique : «c'est sûr, la démonstration, c'est aussi leur en mettre plein la vue », « de temps en temps, les calmer avec une bonne démonstration, c'est pas plus mal... ». Et, cette récurrence semble même parfois dépasser l'espace didactique et paraît plutôt être une source de plaisir narcissique, celle d'être le sujet sachant : " si tu fais un truc et que tu le fais bien, c'est vrai que c'est bien aussi pour toi », « il y a un plaisir à asseoir ton autorité en montrant que tu maîtrises ».

II semble ainsi que l'expérience de l'enseignante marque les différentes formes de prise de responsabilités vis-à-vis de l'émergence du savoir. Sa position symbolique ne pouvant être identiquement assumée, elle ne peut se rattacher aux mêmes procédures ostensives

2 C'est une particularité explicite de l'enseignement de l'EPS. Le professeur d'EPS sans pouvoir toujours mettre en œuvre le savoir en connaît généralement les déterminants structurels. 
pour s'engager dans ces responsabilités. Car la position symbolique du sujet enseignant dans la classe le confronte à des responsabilités qu'il lui est difficile d'assumer ou qu'il ne peut s'empêcher de prendre.

\section{Conclusion : expérience, enseignement et didactique clinique}

Cet article, en présentant le cas d'une enseignante d'EPS débutante, tente de montrer comment le processus de transmission des savoirs et les phénomènes didactiques qu'il donne à décrire peuvent être interprétés à partir d'une prise en compte de la subjectivité du professeur. Celui-ci, fort d'une histoire singulière, d'expériences uniques, est marqué d'un rapport spécifique aux savoirs qu'il a la charge d'enseigner. Et toute cette singularité oriente les phénomènes didactiques de la même manière que les contraintes didactiques pèsent à leur tour sur le sujet inscrit dans le processus de transmission.

La recherche en didactique nous semble actuellement être caractérisée par deux principaux axes de travail. D'une part, elle s'intéresse aux phénomènes transpositifs, c'està-dire à l'évolution et à la transformation des objets de savoir qu'entraîne leur prise dans une intention didactique, du champ scientifique et social jusqu'à leur mise en œuvre dans la classe. D'autre part, le consensus à propos d'une conception de l'enseignement fondée sur la dévolution, sur l'importance de l'activité de l'élève, d'une prise de responsabilités de sa part, oriente la recherche en didactique vers une tentative de compréhension des possibilités et des conditions de fonctionnement de ce processus de transmission. II s'agit de comprendre comment fonctionne le milieu qui porte les objets de savoir ainsi que le jeu qui s'instaure entre l'enseignant et ses élèves à propos de ces objets, dans son inscription temporelle et topologique.

Alors, la didactique clinique permet l'apport de nouveaux éléments de compréhension sur ces deux orientations de recherche. Elle réinterroge les phénomènes transpositifs en identifiant le processus, qui d'une référence sociale la transforme en objets de savoirs enseignés, comme un processus de conversion didactique de l'expérience de l'enseignant. C'est-à-dire que la référence même est réexaminée, considérant que la référence sociale pour entrer dans la classe ne peut plus être que le positionnement singulier du sujet enseignant dans ce champ. L'approche de ce positionnement présentée ici en terme de rapport aux savoirs peut alors permettre de systématiser un des fondements de ce processus. D'autres chercheurs en didactique clinique ont pu déjà tenter de modéliser certains aspects de ce processus, en terme de «filtres » (Loizon, 2004) chez les professeurs de judo, ou de « transposition expérientielle » (Jourdan, 2005) 
chez les étudiants en EPS à l'IUFM. Quant au deuxième axe de travail traité par la didactique, la perspective clinique permet d'éclairer le jeu établi par l'enseignant avec ses élèves à propos des savoirs, à partir des contraintes intrinsèques qui pèsent sur l'enseignant lui-même. Car, inscrit dans le didactique, il occupe une place symbolique, il place son corps comme image sous l'attention des élèves, il se confronte à une toute maîtrise impossible. Ainsi, l'approche du sujet enseignant à partir de son expérience personnelle dans les APSA permet de prendre en compte son histoire singulière avec les objets de savoir, c'est-à-dire l'un des fondements de sa singularité, et de voir comment celle-ci oriente la forme de gestion du contrat didactique. Elle réinterroge par exemple les problématiques de l'institutionnalisation ou de l'ostension des savoirs en repositionnant le sujet enseignant comme responsable et générateur de ces processus.

Le cas présenté ici permet alors, à partir de la problématique de l'expérience personnelle de l'enseignant, l'extraction de «cadres d'analyse » (Héritier, Xanthakou, 2004) susceptibles de constituer une des armatures de la didactique clinique. L'intention n'est pas de juger la qualité des enseignements menés au regard de l'expérience personnelle antérieure qui les soutient, mais bien de constituer une structure conceptuelle capable de décrire, comparer et interpréter les phénomènes didactiques en les dialectisant avec la subjectivité de celui qui les conduit. Ainsi, le processus de conversion didactique, le statut de «sujet supposé savoir » et le rapport à la contingence peuvent s'édifier comme des outils conceptuels capables de situer le cas de chaque enseignant dans des éléments systématiquement constitutifs du didactique.

\section{Bibliographie}

Beillerot, J. (1996). Désir, désir de savoir, désir d'apprendre. In Beillerot, BlanchardLaville, Mosconi (dir.), Pour une clinique du rapport au savoir. Paris : L'Harmattan.

Beillerot, J. (2000). Le savoir, une notion nécessaire. In Mosconi, Beillerot BlanchardLaville (dir.), Formes et formations du rapport au savoir. Paris :L'Harmattan.

Berdot, P., Blanchard-Laville, C., Bronner, A. (2000). Savoir mathématique et rapport au savoir des professeurs de mathématiques. Traumatismes en chaîne et résonances identitaires. In Mosconi, Beillerot Blanchard-Laville (dir.), Formes et formations du rapport au savoir. Paris : L'Harmattan.

Blanchard-Laville, C. (2001). Les enseignants, entre plaisir et souffrance. Paris : PUF. 
Blanchard-Laville, C. (2002). Rapport au savoir : que nous dit la clinique ? In Actes des 3èmes journées franco-québécoises de didactique : Rapport au savoir et didactiques. Sorbonne : Paris.

Brousseau, G. (1986). Théorisation des phénomènes d'enseignement des mathématiques. Thèse d'Etat. Bordeaux, Université Bordeaux 1.

Brousseau, G. (1998). Théorie des situations didactiques. Grenoble : La Pensée Sauvage. Buznic-Bourgeacq, P. (2005). L'expérience de l'enseignant et ses implications didactiques: une étude de cas en EPS. In Actes du $5^{\text {ème }}$ colloque international Recherches et formation. IUFM de Nantes.

Chevallard, Y. (1991). La transposition didactique : du savoir savant au savoir enseigné. Grenoble : La pensée sauvage.

Chevallard,Y. (1989). Le concept de rapport au savoir : rapport personnel, rapport institutionnel, rapport officiel. In Actes du séminaire de didactique. Grenoble: IMAG.

Chevallard Y. (1992). Concepts fondamentaux de la didactique : perspectives apportées par une approche anthropologique. Recherches en didactique des mathématiques, (12/1).

Curie, J., Tap, P. (1983). Rapport d'activité scientifique 1979-1983, Personnalisation et changements sociaux, Laboratoire associé au CNRS, Toulouse.

Héritier, F., Xanthakou, M. (2004). Corps et affects. Paris : Odile Jacob.

Jourdan, I. (2005). L'évolution du rapport au savoir comme "révélateur » des logiques de professionnalisation : études de cas en formation initiale en EPS à l'IUFM de MidiPyrénées. Thèse de doctorat. Toulouse, Université Toulouse 3.

Lacan, J. (1966). Fonction et champ de la parole et du langage. In Ecrits. Paris : Seuil. Laplanche, J., Pontalis, J.-B. (1967). Vocabulaire de la psychanalyse. Paris : PUF.

Loizon, D. (2004). Analyse des pratiques d'enseignement du judo : identification du savoir transmis à travers les variables didactiques utilisées par les enseignants en club et en EPS. Thèse de doctorat. Toulouse : Université Toulouse 3.

Loquet, M., Marsenach, J., Amade-Escot, C. (2005). Théorie des situations didactiques en éducation physique. Pourquoi ? Comment? Quelle transposition? In Salin, Clanché, Sarrazy (Ed.), Sur la théorie des situations didactiques. Grenoble : La pensée sauvage.

Margnes, E. (2002). L'intention didactique dans l'enseignement du judo, des choix culturels d'ordre éthique et technique. Etude de cas de situations didactiques - 
leurs mises en scène - pour des débutants dans la formation initiale en STAPS.

Toulouse, Thèse de doctorat, Université Toulouse 3.

Mosconi, N., Beillerot, J., Blanchard-Laville, C. (2000). Formes et formations du rapport au savoir. Paris : L'Harmattan.

Pineau, G., Legrand, J.-L. (1996). Les histoires de vie. Paris : PUF.

Salin, M.-H. (2002). Les pratiques ostensives d'un enseignement des mathématiques comme objet d'analyse du travail du professeur. In Venturini, Amade-Escot, Terrisse (dir.), Etudes des pratiques effectives : l'approche des didactiques. La Pensée Sauvage.

Sarrazy, B. (1995). Le contrat didactique. Revue française de pédagogie, (112).

Sensevy, G. (1997). Désirs, institutions, savoir. In Blanchard-Laville (dir.), Variations sur une leçon de mathématiques. Paris : L'Harmattan.

Sensevy, G., Quilio, S. (2002). Les discours du professeur. Vers une pragmatique didactique. Revue française de pédagogie, (141).

Terrisse, A. (1994). La question du savoir dans la didactique des activités physiques et sportives : essai de formalisation. Note de synthèse à l'HDR. Toulouse, Université Toulouse 3.

Terrisse, A. (1998). Transposition didactique et prise en compte du sujet : perspectives de recherche en éducation physique et sportive. In Amade-Escot, Barrué, Bos, Dufor, Dugrand, Terrisse (dir.), Recherches en EPS, bilan et perspectives. Paris: Editions Revue EPS.

Terrisse, A. (2000). Epistémologie de la recherche clinique en sports de combat. In Terrisse (dir.), Recherches en sports de combat et en arts martiaux : état des lieux. Paris : Ed. Revue EPS.

Terrisse, A. (2001). La référence dans l'enseignement de l'éducation physique et sportive. In Terrisse (Ed.), Didactique des disciplines, Les références au savoir. De Boëck.

Terrisse, A., Léziart, Y. (1999). Une réponse à la contingence : le contrat didactique de Guy Brousseau. Actes du Congrès de l'AIESEP, Besançon.

Terrisse, A., Loizon, D., Buznic-Bourgeacq, P. (2005). La place du corps dans l'enseignement de l'EPS. In Actes du $5^{\text {ème }}$ colloque international Recherches et formation. IUFM de Nantes.

Venturini, P., Amade-Escot, C., Terrisse, A. (2002). Etudes des pratiques effectives : l'approche des didactiques. La Pensée Sauvage. 